Article

\title{
An Investigation into the Appropriateness of Car-Following Models in Assessing Autonomous Vehicles
}

\author{
Akito Higatani ${ }^{1}$ and Wafaa Saleh ${ }^{2,3, *,+}$ \\ 1 Hanshin Expressway Co., Ltd., 7-15-26, Osaka 553-0003, Japan; higatani-akito@hanshin-exp.co.jp \\ 2 College of Engineering, Princess Nourah Bint Abdulrahman University, Riyadh 84428, Saudi Arabia \\ 3 Transport Engineering, Transport Research Institute, Edinburgh Napier University, 10 Colinton Road, \\ Edinburgh EH10 5DT, UK \\ * Correspondence: w.saleh@napier.ac.uk or wsshoukry@pnu.edu.sa \\ + Visiting Professor.
}

check for updates

Citation: Higatani, A.; Saleh, W. An Investigation into the

Appropriateness of Car-Following Models in Assessing Autonomous Vehicles. Sensors 2021, 21, 7131. https://doi.org/10.3390/s21217131

Academic Editors: Javier Alonso Ruiz and Iván García Daza

Received: 6 September 2021

Accepted: 18 October 2021

Published: 27 October 2021

Publisher's Note: MDPI stays neutral with regard to jurisdictional claims in published maps and institutional affiliations.

Copyright: (c) 2021 by the authors. Licensee MDPI, Basel, Switzerland. This article is an open access article distributed under the terms and conditions of the Creative Commons Attribution (CC BY) license (https:// creativecommons.org/licenses/by/ $4.0 /)$.

\begin{abstract}
The dramatic progress of Intelligent Transportation Systems (ITS) has made autodriving technology extensively emphasised. Various models have been developed for the aim of modelling the behaviour of autonomous vehicles and their impacts on traffic, although there is still a lot to be researched about the technology. There are three main features that need to be represented in any car-following model to enable it to model autonomous vehicles: desired time gap, collision avoidance system and sensor detection range. Most available car-following models satisfy the first feature, most of the available car-following models do not satisfy the second feature and only few models satisfy the third feature. Therefore, conclusions from such models must be taken cautiously. Any of these models could be considered for updating to include a collision avoidance-system module, in order to be able to model autonomous vehicles. The Helly model is car-following model that has a simple structure and is sometimes used as the controller for Autonomous Vehicles (AV), but it does not have a collision avoidance concept. In this paper, the Helly model, which is a very commonly used classic car-following model is assessed and examined for possible update for the purpose of using it to model autonomous vehicles more efficiently. This involves assessing the parameters of the model and investigating the possible update of the model to include a collision avoidance-system module. There are two procedures that have been investigated in this paper to assess the Helly model to allow for a more realistic modelling of autonomous vehicles. The first technique is to investigate and assess the values of the parameters of the model. The second procedure is to modify the formula of that model to include a collision avoidance system. The results show that the performance of the modified full-range Auto Cruising Control (FACC) Helly model is superior to the other models in almost all situations and for almost all time-gap settings. Only the Alexandros E. Papacharalampous's Model (A.E.P.) controller seems to perform slightly better than the (FACC) Helly model. Therefore, it is reasonable to suggest that the (FACC) Helly model be recommended as the most accurate model to use to represent autonomous vehicles in microsimulations, and that it should be further investigated.
\end{abstract}

Keywords: autonomous vehicles; car-following models; Helly models; ITS

\section{Introduction}

The significant increase in technological advances and progress in Intelligent Transportation Systems (ITS) have enabled autodriving and Autonomous Vehicles (AV) technology and their related research to become possible. There are six stages of automation, where full automation, level five, is the final stage, and zero automation is level zero. Full automation means a driverless vehicle. If an autonomous vehicle is equal to a driverless vehicle, then partial autonomous vehicles (say level two or three) are already being sold in the market. There are some core technologies related to a driverless vehicle, such as collision avoidance system, auto steering technology, lane changing technology, intelligent 
parking systems, communication technology and sensor technology. Road-to-vehicle connecting technology such as Intelligent Speed Adaptation (ISA) is implicitly included in the communication technology. Furthermore, platoon technology may be also utilised as a core technology in the field of freight transport.

Many studies and researchers have investigated issues related to numerous aspects of autonomous vehicles. These include social and technological issues, and prediction of the market. Various models have been developed to model Autonomous Vehicles (AV), yet most of them are still using more or less the same principles of the models that have been developed for manually driven vehicles and driving behaviour. Therefore, conclusions from such models have to be taken cautiously. There is a great deal still unknown about this technology and its impacts on traffic behaviour and transportation systems. Car-following models are typically used as methods to investigate driving behaviour and the impact of the behaviour of the lead vehicle on the behaviour of the following vehicle. Various car-following models have been developed since the 1950's to represent driver's car-following behaviour such as the Gipps Model (GM), Collision Avoidance Model (CA model), Helly model, Optimal Velocity Model (OVM), Intelligent Driver Model (IDM) and Intelligent Driver Model+ (IDM+). Most of these models have also been used to model the AV behaviour, with or without modifications.

The three main features of autonomous vehicles that are considered in this research are: (1) Auto Cruise Control technology. This is by far the most important technology in autonomous, driverless vehicles. Auto Cruise Control (ACC) technology and its full-range Auto Cruise Control (FACC) have been around for the past 60 years, at least. ACC is mainly a technology which adjusts the gap and sustains the desired gap using sensors. This means that the vehicle is able to maintain constant distance gap as well as constant speed, which results in reducing chances of interrupting flowing traffic; (2) The second feature of an AV is the collision avoidance system. Since all autonomous vehicles, in reality, will have this feature, autonomous vehicle controllers and models should have such a function. Any models that are used to represent $\mathrm{AV}$ appropriately should be verified regarding whether they have an appropriate braking function; and (3) a sensor detection range. A precise and fast sensor detection system is vital for autonomous vehicles and autodriving. This is important to be able to identify vehicles' shapes, distances, speeds and movements.

To conclude, for any model to be appropriately used to model behaviour of AV and their behaviour, it needs to satisfy the three requirements: the desired time gap, collision avoidance system and sensor detection range. Some models have limitations in representing varying desired gaps that depend on vehicle speed. For example, the GM model does not have a term for desired time gap or desired gap. Therefore, it cannot represent AV behaviour and it will have to be adjusted with the right parameters that depend on the speed. The four car-following models and controllers that will be investigated in this study all have a desired time-gap feature. These are the Helly model, IDM, IDM+ and Alexandros E. Papacharalampous's Model (A.E.P.) controller.

This study reports on an investigation of the appropriateness of car-following models in replicating the behaviour of autonomous vehicles. Data from vehicles that are equipped with full-range ACC (FACC) and are already commercially available in the market were obtained and utilised in the assessment. The Helly model, which is a very frequently used car-following model, is assessed and examined for appropriateness for update for the purpose of using it to model autonomous vehicles more efficiently. This involved assessing the parameters of the model and investigating a possible update to include a collision avoidance system component. An enhanced car-following model, Helly (FACC), is proposed, assessed and validated. The validation included a comparative analysis of models' performance in two paradigms that are taken from the Japanese collision avoidance-system standards. These are: a paradigm that states that "when the following vehicle approaches at $50 \mathrm{~km} / \mathrm{h}$ to a stationary vehicle, the following vehicle should not collide with the stationary vehicle or the following vehicle's speed should be less than $20 \mathrm{~km} / \mathrm{h}$ if a collision occurs". 
The paper is structured as follows. Section 2 presents previous related work. Discussions of car-following models and their appropriateness for modelling AV are also presented. Section 3 is devoted to the investigation of the appropriateness of the Helly model to represent AV. In this Section, the parameters of the model are tested and examined using case scenarios. In addition, modifying the model formulation as well as validation of the results are also presented in Section 3. Section 4 is devoted to the conclusions and further research recommendations.

\section{Previous Related Work}

Microsimulation modelling and car-following models are typically used as methods to investigate the impact of autonomous vehicles' operation and monitoring [1-8]. Various carfollowing models have been developed since the 1950's to represent driver's car-following behaviour such as the Gipps Model (GM) [4], collision avoidance model (CA model) [4], Helly model [5], Optimal Velocity Model (OVM) [6], Intelligent Driver Model (IDM) [7], and IDM+ [8].

Research on autonomous vehicles using microsimulation can be divided into two types. One is research using microsimulation with existing car-following models such as IDM and IDM+ [9]. Martin Treiber et al. [7] reproduced driving behaviour of autonomous vehicles using IDM and investigated the impact of autonomous vehicles on traffic flow [9]. Interestingly, they reported in the paper that human and automated driving behaviours are fundamentally different in terms of reaction time and driving strategies. The IDM can be calibrated to actual traffic data, but these two effects are not significantly affecting the results. Suzuki et al. [10] also represented driving behaviour of autonomous vehicles using IDM+ with different parameters representing human drivers. The second type of study reported the results of using Auto Cruise Controllers (ACC) to represent autonomous vehicles' behaviour [11]. This type of research is more common in characterising travel behaviour than that of car-following models. The history of the ACC controllers is relatively new. Many ACC controllers have been developed based on simple structures, such as that of Wouter et al. [8] who used a Connected Auto Cruising Control (CACC) controller to investigate the possibility of dampening shock waves. Although various car-following models and ACC controllers have been used in many research works, there is not much evidence of a clear superiority of any of these models in terms of representation of autonomous vehicles. In the following section, work related to car-following models and ACC are reviewed and assessed in terms of their appropriateness for modelling AV.

\subsection{Car-Following Models}

Car-following models are microsimulation models and tools that are used to predict the longitudinal acceleration of a vehicle based on the speed and position of a leading vehicle in a traffic lane. The Helly model is a car-following model; the vehicle acceleration is controlled by the distance gap and speed difference with the following algorithm in Equation (1):

$$
\frac{d v_{i}(t)}{d t}=\alpha \Delta v_{i}(t)+f i\left(\Delta x_{i}(t)-\left(s_{0}+v_{i}(t) T\right)\right)
$$

where:

$i$ denotes the vehicle index

$v_{i}(t)$ is speed

$\Delta v_{i}(t)$ is relative speed with respect to the preceding vehicle

$\Delta x_{i}(t)$ is distance gap

$T$ is desired time gap

$s_{0}$ is the minimum gap at standstill

$\alpha$ is the sensitivity parameter with respect to relative speed $\Delta v_{i}(t)$, and

$\beta$ is the sensitivity parameter with respect to the difference between the current gap and the desired gap. 
Moreover, the advanced version of this Helly model-type controller is formulated as follows (Equation (2)) [8,12]:

$$
\frac{d v_{i}(t)}{d t}=\min \left[k\left(v_{0}-v_{i}(t)\right),\left(f f \Delta v_{i}(t)+f i\left(\Delta x_{i}(t)-\left(s_{0}+v_{i}(t) T\right)\right)\right]\right.
$$

where $v_{0}$ is desired speed and $k$ is the sensitivity with respect to the difference between the desired speed and the current speed.

The reason for adding the first formula is to distinguish between free driving behaviour and following behaviour. These Helly model-type controllers have no collision avoidance concept, so they are not able to represent braking behaviour adequately, such as emergency stopping. Other types of car-following models are available. These include for example the Intelligent Driver Model (IDM), IDM+ and Alexandros E. Papacharalampous's Model (A.E.P.) which have been assessed in this study. Arne Kesting et al. [11] suggested that the IDM-type controller whose parameters are different from those of nonautonomous vehicles are best to use. The controller takes different parameters which include the desired time gap according to the traffic state such as 'Free flow, 'Congested traffic', etc. The acceleration in this model is given in Equation (3) by:

$$
\begin{aligned}
\frac{d v_{i}(t)}{d t}=\lambda_{a} a[(1- & \left.\left.\left(\frac{v_{i}(t)}{v_{0}}\right)^{4}-\left(\frac{s^{*}\left(v_{i}(t), \Delta v_{i}(t)\right)}{\Delta x_{i}(t)}\right)^{2}\right)\right] s^{*}\left(v_{i}(t), \Delta v_{i}(t)\right)=s_{0}+v_{i}(t) \lambda_{T} T+\frac{v_{i}(t) \Delta v_{i}(t)}{2 \sqrt{\lambda_{a} a \lambda_{b} b}} \\
& \text { where: } \\
& a \text { is the maximum acceleration } \\
& b \text { is the desired acceleration and } \\
& \lambda_{a}, \lambda_{b}, \lambda_{T} \text { are coefficients represent the multiplication factors }
\end{aligned}
$$

A feature of IDM [6] and IDM+ [7] is the nonlinear response to speed differences. These models are quite robust and user-friendly in simulating human driver behaviour. However, these models still have limitations in representing autonomous vehicle behaviour. Additionally, there is another type of controller that is based on the design of a flexible Model Predictive Controller, as presented in Equation (4) [13-15].

$$
\frac{d v_{i}(t)}{d t}=\left\{\begin{array}{c}
k_{1}\left(v_{\text {input }, i}-v_{i}(t)\right)+k_{2} \frac{\Delta v_{i}(t)}{\Delta x_{i}(t)}, \quad \text { if } \Delta x_{i}(t) \leq \gamma^{A C C} \\
k_{1}\left(v_{0}-v_{i}(t)\right), \quad \text { if } \Delta x_{i}(t) \geq \gamma^{A C C}
\end{array} \quad v_{\text {input }, i}=\min \left(\frac{\Delta x_{i}(t)-s_{0}}{T}, v_{0}\right)\right.
$$

with

$\Delta x_{i}(t)>0$ (non - collision constraint)

$0 \leq v_{i}(t) \leq v_{\max }$ (physical speed range)

$a_{\min } \leq \frac{d v_{i}(t)}{d t} \leq a_{\max }$ (admissible acceleration range)

where:

variable $v_{\text {input }, i}$ is a gap-dependent desired speed

$\gamma^{A C C}$ is the onboard sensor detection range

$k_{1}$ and $k_{2}$ are feedback gains

A feature of this model is that the sensor detection range is clearly defined. This concept implies that the behaviour differs between within the sensor detection range and out of the range.

\subsection{Appropriateness of Car-Following Models for Modelling AV}

Table 1 below shows the most frequently used car-following models and their capabilities to replicate the behaviour of autonomous vehicles. There are three main requirements that need to be satisfied for these models to be useful in representing behaviour of autonomous vehicles. These are: the desired time gap, collision avoidance system and sensor detection range. Some models have limitations in representing varying desired gaps that 
depend on vehicle speed. For example, the GM model does not have the term of desired time gap or desired gap. Therefore, it cannot represent AV behaviour and it will have to be adjusted with the right parameters that depend on the speed. There are four car-following models and controllers that have a desired time-gap feature. These are Helly model, IDM, IDM+ and A.E.P. controller. Secondly, most autonomous vehicles are equipped with collision avoidance systems.

Table 1. The main features of autonomous vehicles that need to be represented in models.

\begin{tabular}{cccc}
\hline $\begin{array}{c}\text { Car-Following Model } \\
\text { or ACC Controller }\end{array}$ & $\begin{array}{c}\text { Desired Time } \\
\text { Gap or Desired Gap }\end{array}$ & $\begin{array}{c}\text { Collision Avoidance } \\
\text { System }\end{array}$ & $\begin{array}{c}\text { Sensor Detection } \\
\text { Range }\end{array}$ \\
\hline GM model & X & $X$ & $X$ \\
\hline Collision Avoidace (CA) model & X & $X$ & $X$ \\
\hline Helly model & $\checkmark$ & $X$ & $X$ \\
\hline Optimal Velocity Model (OVM) & $X$ & $X$ & $X$ \\
\hline Intelligent Driver Model (IDM) & $\checkmark$ & $X$ & $X$ \\
\hline Intelligent Driver Model (IDM)+ & $\checkmark$ & $X$ & $\checkmark$ \\
\hline $\begin{array}{c}\text { Controller proposed } \\
\text { by A. E. Papacharalampous }\end{array}$ & $\checkmark$ & $X$ & $\checkmark$ \\
\hline
\end{tabular}

$\checkmark:$ The feature is represented in model. X: The feature is not represented in model.

Therefore, controllers that are used to model AV should have such a function to represent collision avoidance behaviour. Any of these models could be considered for update in terms of collision avoidance systems in order to be able to model the control of autonomous vehicles. Finally, most car-following models do not have the concept of sensor detection range, only the ACC controller proposed by Alexandros E. Papacharalampous et al. [14] (hereinafter this is referred to as A.E.P. controller) has that concept.

In conclusion, as mentioned above, various ACC controllers have been developed. The simplest controller is the Helly model-type controller, which has a classic structure and is sometimes used as the controller for Autonomous Vehicles (AV), but has no collision avoidance concept. Therefore, it is not capable of representing braking behaviour, such as emergency stopping. In this study, the Helly model is investigated for update to include a collision avoidance system module as discussed in the following Sections.

\section{Helly Model Investigation}

In this Section, the Helly model will be investigated for its appropriateness for modelling autonomous vehicles. Two procedures that will be utilised in this paper to allow more realistic modelling of autonomous vehicles include: an examination of the model parameters, and then an update of the model to include the collision avoidance system.

\subsection{Assessment of Model's Parameters}

The Helly model is presented by Equation (1) above. There are two coefficients of the model; $\alpha$ which is the sensitivity parameter with respect to relative speed $\Delta v_{i}(t)$, and $\beta$ is the sensitivity parameter with respect to the difference between the current gap and the desired gap. Examining these coefficients is illustrated here by an example as presented in Figure 1. In this case, the leading vehicle and the following vehicle are both driving with a speed of $10 \mathrm{~km} / \mathrm{h}$ and the first gap is $5.25 \mathrm{~m}$. The leading vehicle is decelerating with a rate of $0.5 \mathrm{~m} / \mathrm{s}^{2}$ from $5 \mathrm{~s}$ to $10 \mathrm{~s}$, and then it accelerates with a rate of $0.5 \mathrm{~m} / \mathrm{s}^{2}$ from $15 \mathrm{~s}$ to $20 \mathrm{~s}$. The desired time gap was calculated using a very short setting of the time gap control strategy. The values of $\alpha$ and $\beta$ are varied from 0.125 to 1 , as illustrated in Figures 2 and 3 below. 


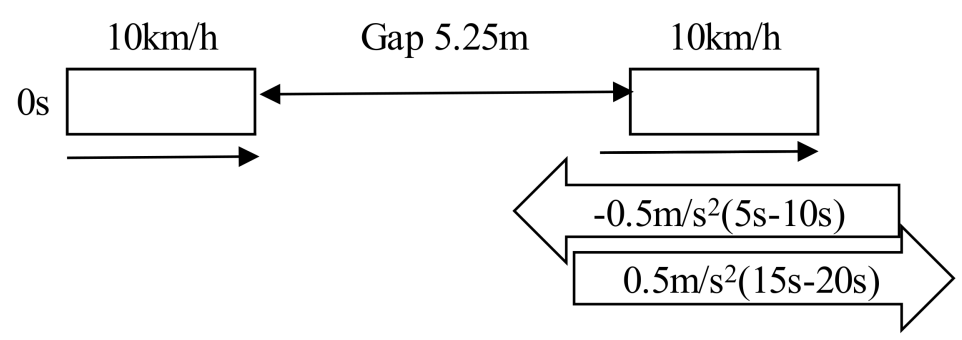

Figure 1. Representation of the following behaviour (example case).

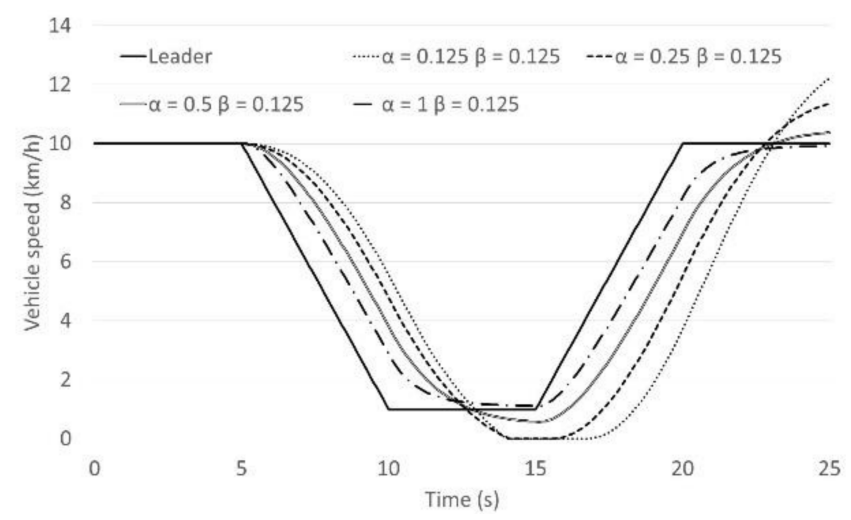

Figure 2. The following vehicle's speed behaviour ( $\alpha$ is fixed).

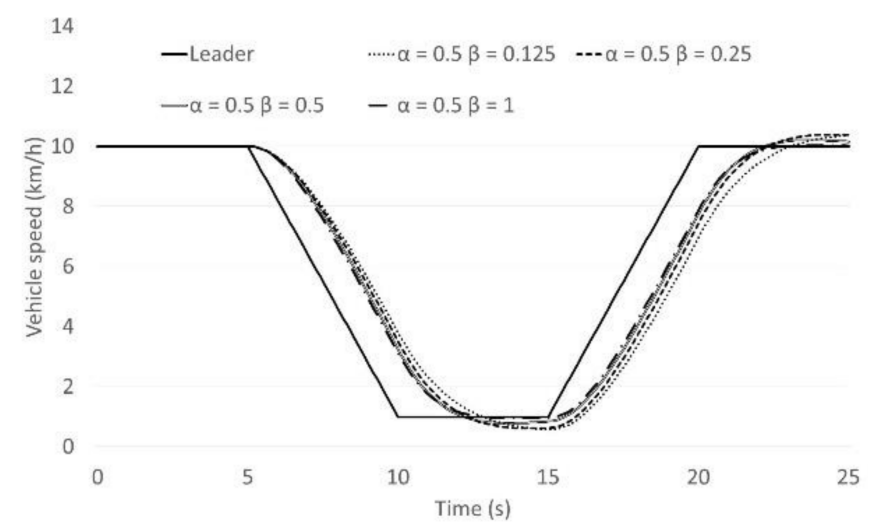

Figure 3. The following vehicle's speed behaviour ( $\beta$ is fixed).

Figures 2 and 3 represent the following vehicle speed transition. From the results it appears that $\alpha$ has a much bigger impact on the car-following behaviour than $\beta$. Additionally, for values of $\alpha$ smaller than 0.5 , such as 0.25 or 0.125 , the following vehicle will not be able to follow the leading vehicle smoothly in congested flow. This tendency appears in the summarised parameter result of the Helly model [16]. Many researchers have tended to adjust the $\beta$ coefficient but not the $\alpha$ coefficient.

The next step is to assess the impact of the $\beta$ coefficient on braking behaviour when another vehicle cuts in the gap. The example case is shown in Figure 4. The leading vehicle and following vehicle were driving at $60 \mathrm{~km} / \mathrm{h}$ and the first gap was $20 \mathrm{~m}$. Then, another vehicle cuts in the gap. The values of $\beta$ were varied between 0.125 and 1 as shown in Figures 5 and 6. Figure 5 shows the results of the following vehicle's speed transition. Figure 6 shows the results of the following vehicle's acceleration transition. According to the results, if the value of $\beta$ is over 0.25 , the following vehicle decelerates at a rate from $0.3 \mathrm{G}$ to $1.3 \mathrm{G}$ when another vehicle cuts in the gap. In other words, the following vehicle will need to make a fierce brake despite the fact that it is not actually a dangerous situation. That behaviour might cause rear-end collisions with the second or third following vehicles and results in unsafe and uncomfortable passenger experiences. In summary, in order to 
improve braking function of the Helly model, it seems that the original parameter values $(\alpha=0.5$ and $\beta=0.125)$ as were suggested by Helly, are best to use in the model.
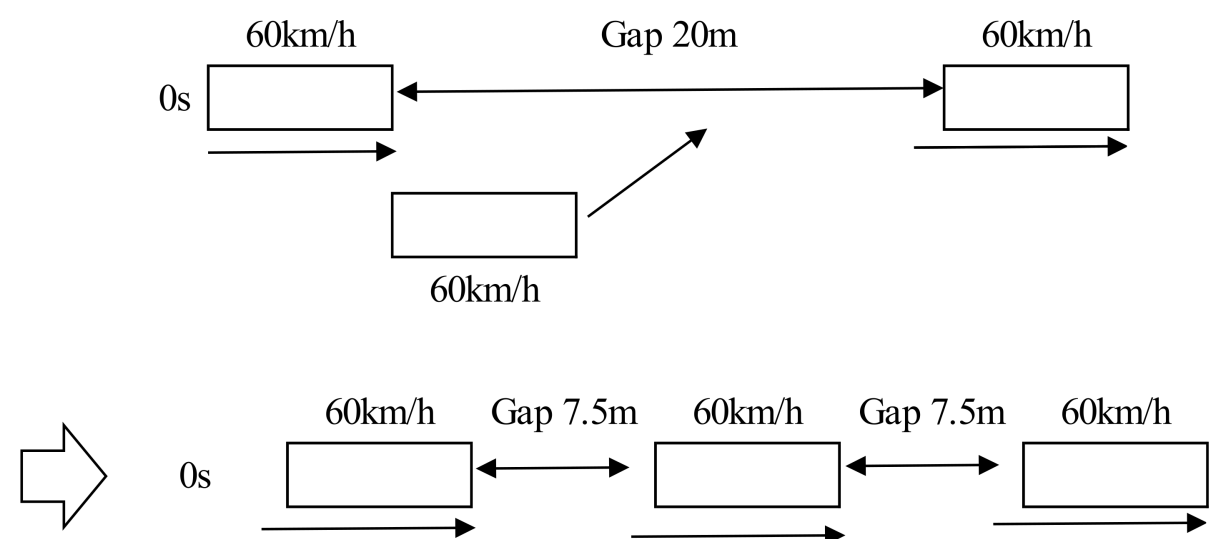

Figure 4. Representation of the cutting off behaviour (example case).

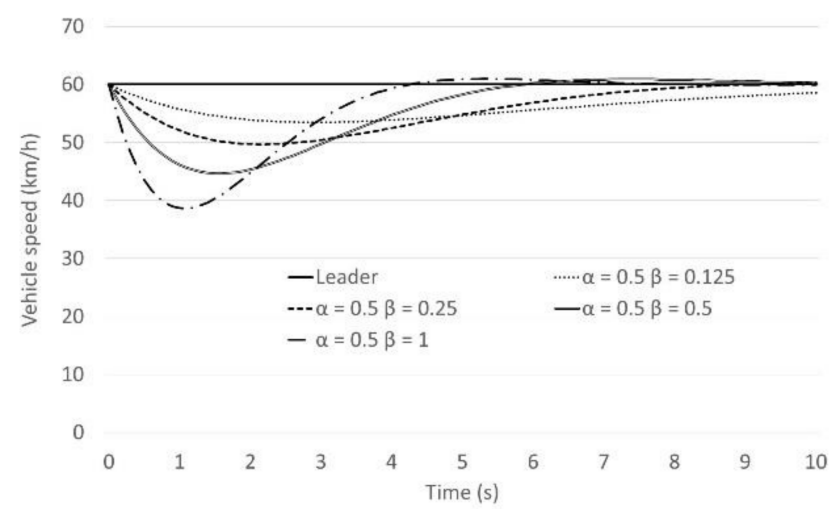

Figure 5. The following vehicle's speed behaviour: Representation of the cutting off behaviour Speed ( $\alpha$ is fixed).

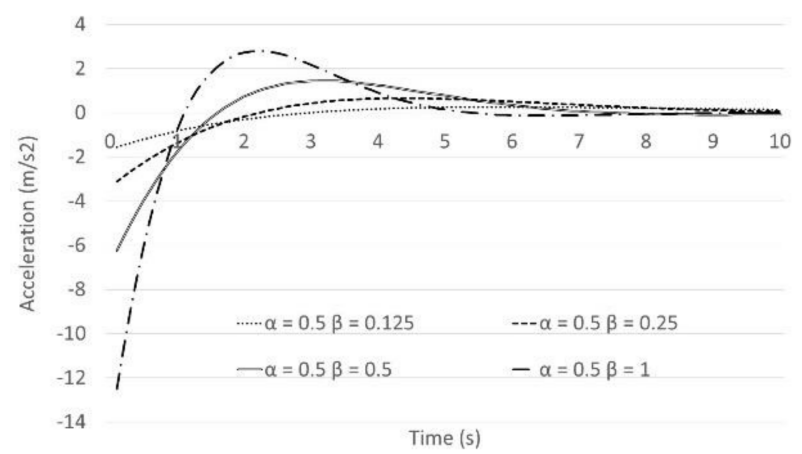

Figure 6. The following vehicle's acceleration behaviour: Representation of the cutting off behaviour speed ( $\alpha$ is fixed).

\subsection{Modifying the Model Formulation}

\subsubsection{Time-Gap Control Strategy}

Most of the controllers for autonomous vehicles have been developed based on timegap control policy and have the term of desired time gap $T$ or $t_{d}$ in their formulas. While setting various desired time gaps is possible in reality, in many of the controllers, time gap is given as a constant value for simplicity. However, it is not very realistic to represent the behaviour of autonomous vehicles using a constant time gap. On the other hand, some of the controllers vary the desired time gap depending on the traffic conditions around the controlled vehicle [11,14]. Arne Ketsting et al. [11] proposed that the desired time gap 
should be halved in congested flows; therefore $\lambda_{T}$ was suggested by them to be set to 0.5 . While this assumption might be effective in reducing congestion, it might not be possible to justify why all drivers have to opt to that choice. Some drivers who would not take a risky approach would possible rather choose to reduce the time gap values. If FACC is an extension of the traffic safety technology of lightening the burden of drivers, people expect that the desired time gap becomes bigger in congested flow because the distance gap is shorter than it is in free flow. Moreover, people would naturally expect that the desired time gap becomes smaller so as to prevent unnecessary cutting-in in free flow traffic.

This study uses data from vehicles that are equipped with FACC and are already commercially available in the market, to assess the performance of car-following models and ACC controllers. Data on the standard gaps that have been obtained from 15 car manuals were attained and are presented in Table 2 below. Drivers who use FACC functions can select a setting for the desired gap range, such as long, middle or short according to the traffic situation as well as their own preferences. The number of settings and the length of distance gaps are not standardised among car manufactures. According to Toyota's manual for example, the number of settings is three [17-19]. If a driver selects the middle setting, the autonomous vehicle will try to maintain a $40 \mathrm{~m}$ gap when the car drives at $80 \mathrm{~km} / \mathrm{h}$. Most of the manuals provide values of gaps at a vehicle high-speed range $(80 \mathrm{~km} / \mathrm{h}$ or $100 \mathrm{~km} / \mathrm{h}$ ). However, only Subaru's manuals provide gap values at other values $(40 \mathrm{~km} / \mathrm{h}$ and $100 \mathrm{~km} / \mathrm{h}$ ).

Table 2. Desired gap of FACC function.

\begin{tabular}{|c|c|c|c|c|c|c|c|c|c|c|c|c|}
\hline \multirow{2}{*}{\multicolumn{2}{|c|}{$\begin{array}{c}\text { Car } \\
\text { Manufacturer. } \\
\text { Gap No. }\end{array}$}} & \multirow{2}{*}{$\begin{array}{c}\text { Toyota } \\
1\end{array}$} & \multicolumn{2}{|c|}{ Honda } & \multicolumn{2}{|c|}{ Nissan } & \multicolumn{2}{|c|}{ Mazda } & \multicolumn{4}{|c|}{ Subaru } \\
\hline & & & 2 & 3 & 4 & 5 & 6 & 7 & 8 & 9 & 10 & 11 \\
\hline \multirow{5}{*}{ 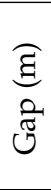 } & very long & & 61 & 78 & & & & & & & & \\
\hline & long & 50 & 47 & 59 & 60 & 60 & 50 & 50 & 30 & 60 & 30 & 60 \\
\hline & middle & 40 & 33 & 40 & 45 & 50 & 40 & 40 & 25 & 50 & 22 & 45 \\
\hline & short & 30 & 25 & 30 & 30 & 40 & 30 & 30 & 20 & 40 & 15 & 30 \\
\hline & very short & & & & & & 25 & 20 & 15 & 30 & & \\
\hline \multicolumn{2}{|r|}{ Speed } & $\begin{array}{c}80 \\
\mathrm{~km} / \mathrm{h}\end{array}$ & $\begin{array}{c}80 \\
\mathrm{~km} / \mathrm{h}\end{array}$ & $\begin{array}{c}100 \\
\mathrm{~km} / \mathrm{h}\end{array}$ & $\begin{array}{c}100 \\
\mathrm{~km} / \mathrm{h}\end{array}$ & $\begin{array}{c}100 \\
\mathrm{~km} / \mathrm{h}\end{array}$ & $\begin{array}{c}80 \\
\mathrm{~km} / \mathrm{h}\end{array}$ & $\begin{array}{c}80 \\
\mathrm{~km} / \mathrm{h}\end{array}$ & $\begin{array}{c}40 \\
\mathrm{~km} / \mathrm{h}\end{array}$ & $\begin{array}{c}100 \\
\mathrm{~km} / \mathrm{h}\end{array}$ & $\begin{array}{c}40 \\
\mathrm{~km} / \mathrm{h}\end{array}$ & $\begin{array}{c}100 \\
\mathrm{~km} / \mathrm{h}\end{array}$ \\
\hline & vehicle type & $\begin{array}{c}\text { Alphard } \\
(2018.3) \\
\text { crown majesta } \\
(2017.5) \\
\text { prius }(2018.3) \\
\end{array}$ & \multicolumn{2}{|c|}{ legend (2018) } & \multicolumn{2}{|l|}{$\begin{array}{l}\text { Cima } \\
(2017.6) \\
\text { fuga } \\
(2017.11)\end{array}$} & \multicolumn{2}{|l|}{$\begin{array}{c}c x-8 \\
(2018.3) \\
c x-5 \\
(2018.2)\end{array}$} & \multicolumn{2}{|c|}{$\begin{array}{l}\text { legacy }(2017.10) \\
\text { impreza }(2017.10) \\
\quad \text { xv }(2017.5)\end{array}$} & \multicolumn{2}{|c|}{$\begin{array}{l}\text { levorg } \\
(2017.8) \\
\text { forester } \\
(2017.4)\end{array}$} \\
\hline
\end{tabular}

Therefore, in this paper, time-gap control strategies to control autonomous vehicles are estimated based on Subaru's manuals (gaps No.8 and No.9 in Table 2). From Table 2, it is clear that the time-gap values at $40 \mathrm{~km} / \mathrm{h}$ are different to those values at $100 \mathrm{~km} / \mathrm{h}$. In terms of the desired gaps, car manuals state that when a vehicle stops, the distance gap will depend on the traffic condition regardless of the desired gap setting. Hence, when a vehicle stops, the desired gap was set at a value of $2 \mathrm{~m}$ regardless of the setting. The time-gap control strategy was estimated as presented in Figure 7 . These values were estimated using the desired gaps at three levels of speed $(0 \mathrm{~km} / \mathrm{h}, 40 \mathrm{~km} / \mathrm{h}$ and $100 \mathrm{~km} / \mathrm{h})$. These values are converted into the desired time gaps and then simply interpolated. 


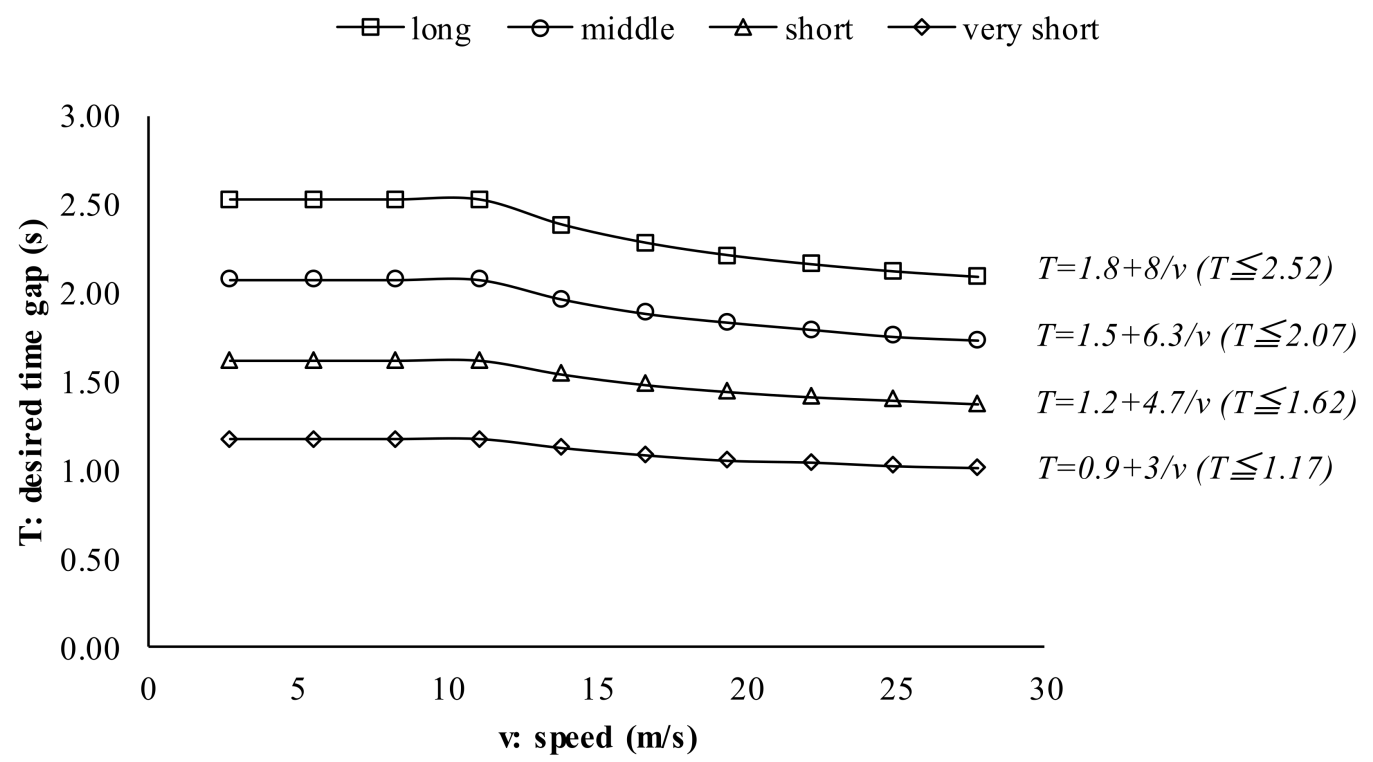

Figure 7. Time-gap control strategy.

\subsubsection{The Updated Model}

In this section we present the updated Helly model which is referred to here as Helly (FACC) model. In this case, the Helly model has been updated to deal with the collision avoidance-system problem. The concept of sensor detection range, the constraints of speed and acceleration and enhancing brake function were introduced into this model as presented in Equation (5) below.

The Helly (FACC) model is formulated as follows:

$$
\begin{gathered}
\frac{d v_{i}(t)}{d t}=\left\{\begin{array}{cc}
\delta\left(\alpha \Delta v_{i}(t)+\beta\left(\Delta x_{i}(t)-\left(s_{0}+v_{i}(t) T(t)\right)\right)\right) & \text { if } \Delta x_{i}(t) \leq s_{i}^{A C C} \\
\gamma\left(v_{0}-v_{i}(t)\right) & \text { if } \Delta x_{i}(t)>s_{i}^{A C C}
\end{array}\right. \\
T(t)=\min \left(k_{1, \text { setting }}+k_{2, \text { setting }} / v_{i}(t), k_{3, \text { setting }}\right)
\end{gathered}
$$

with

$0 \leq v_{i}(t) \leq v_{\max }$

$a_{\min } \leq \frac{d v_{i}(t)}{d t} \leq a_{\max }$

where:

$\alpha$ is the sensitivity parameter with respect to relative speed $\Delta v_{i}(t)$, and equals 0.5 ,

$\beta$ is the sensitivity parameter with respect to the difference between the current gap and the desired gap and equals 0.125 ,

$\gamma$ is the onboard sensor detection range and equals 0.2 ,

$s_{i}^{A C C}$ is the minimum gap at standstill and equals $120 \mathrm{~m}$,

$a_{\min }$ is minimum acceleration and equals $-8 \mathrm{~m} / \mathrm{s}^{2}$ and

$a_{\max }$ is maximum acceleration and equals $0.6 \mathrm{~m} / \mathrm{s}^{2}$,

$\delta$ is defined as the safety risk

The values of the parameters that are related to the time-gap control strategy, $k_{1 \text {, setting, }}$ $k_{2, \text { setting }}$ and $k_{3, \text { setting, }}$ are given in Table 3 . 
Table 3. Values of $k_{1, \text { setting }}, k_{2, \text { setting }}$ and $k_{3, \text { setting }}$.

\begin{tabular}{cccc}
\hline Setting & $\boldsymbol{K}_{\mathbf{1} \text {,setting }}$ & $\boldsymbol{K}_{\mathbf{2} \text {,setting }}$ & $\boldsymbol{K}_{\mathbf{3}, \text { setting }}$ \\
\hline Very short & 1.8 & 8.0 & 2.52 \\
Short & 1.5 & 6.3 & 2.07 \\
Middle & 1.2 & 4.7 & 1.62 \\
Long & 0.9 & 3.0 & 1.17 \\
\hline
\end{tabular}

In the above model, Equation (5) controls the acceleration of the vehicle, Equation (6) represents the time gap control strategy. These equations are the same equations as in the original Helly model. Equation (7) is added to account for the collision avoidance system in an attempt to enhance the brake function of the model. In this case, the $\delta$ parameter is defined as the safety risk, which is introduced in this model as a function of gap settings, $v_{i}$, the speed and $\Delta x_{i}(t)$ is the distance gap, $b_{i}$ and $b_{i-1}$ are decelerations equal to $0.3 \mathrm{G}\left(2.97 \mathrm{~m} / \mathrm{s}^{2}\right)$ and $c$ is $4 \mathrm{~m}$. This concept of the safety risk is based on the Mazda algorithm [17,18], and is illustrated in Equations (8) and (9) and in Figure 8.
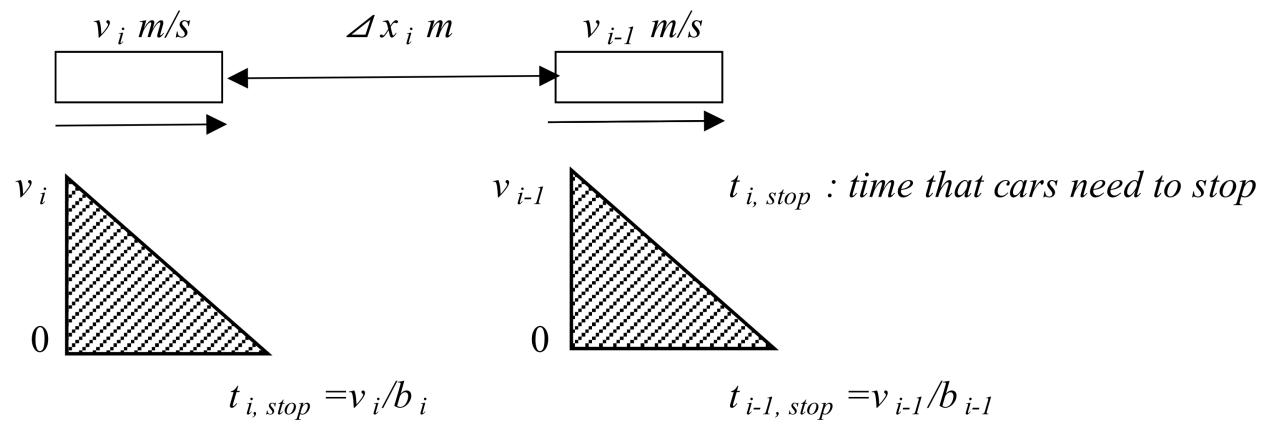

Figure 8. Representation of concept of safety risk.

This function works especially well when a vehicle is in dangerous situation (e.g., emergency stop). On the other hand, the function does not work when a vehicle cuts into the short gap in front of the controlled vehicle. In this case, in order for the vehicle to stop safely, we have:

$$
\frac{v_{i-1} t_{i-1, \text { stop }}}{2}+\Delta x_{i}>\frac{v_{i} t_{i, \text { stop }}}{2}+c
$$

Which leads to:

$$
1>\frac{1}{\Delta x_{i}}\left(\frac{v_{i}^{2}}{2 b_{i}}-\frac{v_{i-1}^{2}}{2 b_{i-1}}+c\right)
$$

where:

$t_{i}$, time that vehicle needs to stop

$b_{i}, b_{i-1}$ are decelerations $\left(\mathrm{m} / \mathrm{s}^{2}\right)$ and

$c$ is the desired safe distance $(\mathrm{m})$

\subsubsection{Model Validation}

To validate the updated model, the braking behaviour of five models, the Helly model, Helly (FACC) model, IDM, IDM+ and A.E.P. controllers have been assessed and compared. The purpose in this case is to investigate how the models perform in terms of meeting the standards of the collision avoidance systems. Data from the Japanese collision avoidance system standards have been utilised in order to set some reference values for the collision avoidance system. The Japanese government publishes standards of collision avoidance systems. These standards have three sets of paradigms; the first paradigm states that "when the following vehicle approaches at $50 \mathrm{~km} / \mathrm{h}$ to a stationary vehicle, the following vehicle should not collide with the stationary vehicle or the following vehicle's speed should be less than $20 \mathrm{~km} / \mathrm{h}$ if a collision occurs". The second paradigm states that "when 
the following vehicle approaches at $50 \mathrm{~km} / \mathrm{h}$ to a leading vehicle that is driving at $20 \mathrm{~km} / \mathrm{h}$, no collision should occur". The third paradigm states that "an alarm to inform drivers of the existence of a vehicle which the vehicle may collide with has to set at least $0.8 \mathrm{~s}$ before the collision avoidance system works".

In this Section, the braking behaviour of the five models (Helly model, Helly (FACC), IDM, IDM+ and A.E.P. controller) are assessed and compared using the first and second sets of the Japanese standards of collision avoidance systems, as presented in Table 4 . In this case, the parameter values of the Helly model, $\alpha$ and $\beta$, are assumed to be the same as those of the updated Helly (FACC) model which are 0.5 and 0.125 . The parameter values of IDM and IDM+ are assumed to be $a=0.6, b=2.8$ and $v_{0}=60 \mathrm{~km} / \mathrm{h}$, based on the work carried out by Suzuki et al. [10] representing the current autonomous vehicle's performance. The parameter values of the A.E.P. controller are assumed to be: $\gamma^{A C C}=120 \mathrm{~m}, k_{1}=0.2$ and $k_{2}=15$ as reported by $[20,21]$.

Table 4. Validation of collision avoidance system.

\begin{tabular}{ccccccc}
\hline Standard & Situation & $\begin{array}{c}\text { Time Gap } \\
\text { Setting }\end{array}$ & $\begin{array}{c}\text { Follower } \\
\mathbf{( k m} / \mathbf{h})\end{array}$ & $\begin{array}{c}\text { Leader } \\
\mathbf{( k m} / \mathbf{h})\end{array}$ & $\begin{array}{c}\text { First Gap } \\
\mathbf{( m )}\end{array}$ & $\begin{array}{c}\text { Behaviour of } \\
\text { Follower }\end{array}$ \\
\hline No.1 & Emergency stop & Very short & 50 & 0 & 17.5 & Stop \\
\hline No.2 & Emergency stop & Very short & 50 & 20 & 17.5 & $\begin{array}{c}\text { Decelerate }\left(-2.97 \mathrm{~m} / \mathrm{s}^{2}(0.3 \mathrm{G})\right) \\
\text { from } 0 \mathrm{~s}-1.8 \mathrm{~s}\end{array}$ \\
\hline
\end{tabular}

The first gap that was calculated using the desired time gap at speed of $50 \mathrm{~km} / \mathrm{h}$ is $17.5 \mathrm{~m}$. Figures 9 and 10 show the following vehicle speed and gap transition of case No.1, while Figures 11 and 12 show the following vehicle speed and gap transition of case No.2. From the results, the four models and controller meet the standards of collision avoidance systems. Only the Helly model does not; it shows a collision rather than maintaining a gap similar to the other models (Figures 10 and 12 for both cases). This is because the Helly model does not have a collision avoidance function in it.

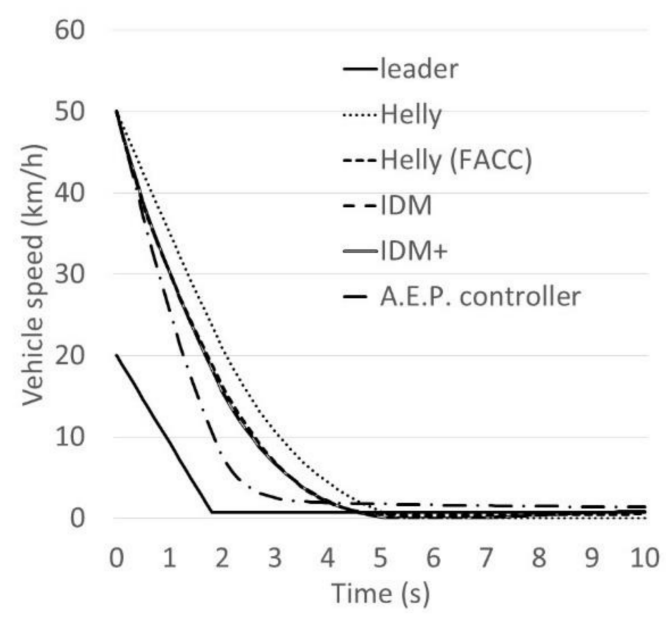

Figure 9. The following vehicle's speed transition of case No.1. 


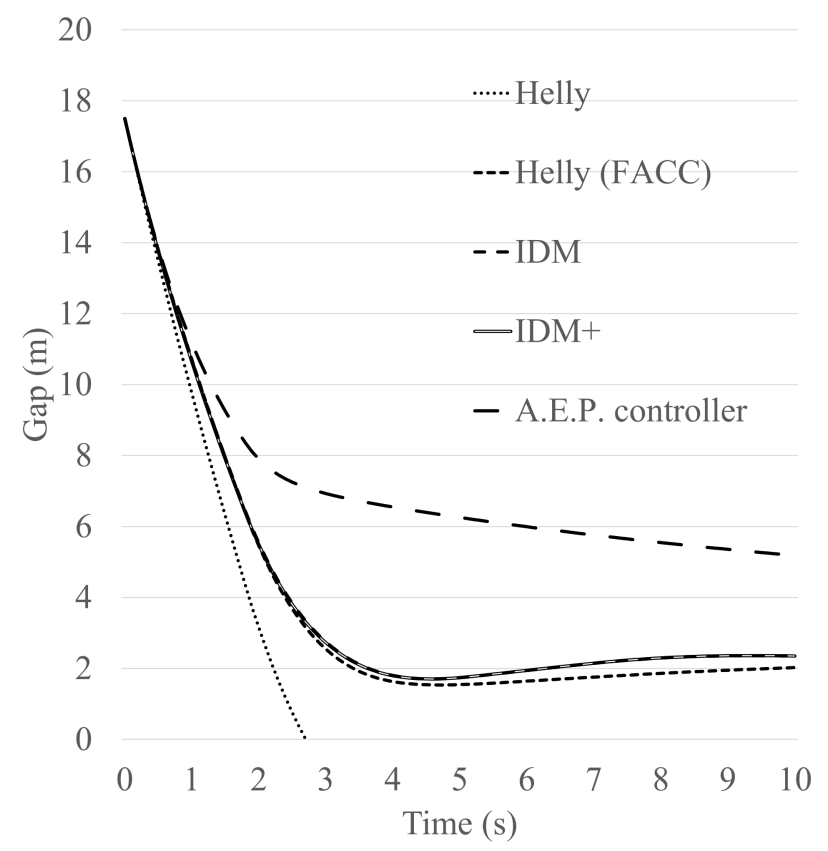

Figure 10. The following vehicle's gap transition of case No.1.

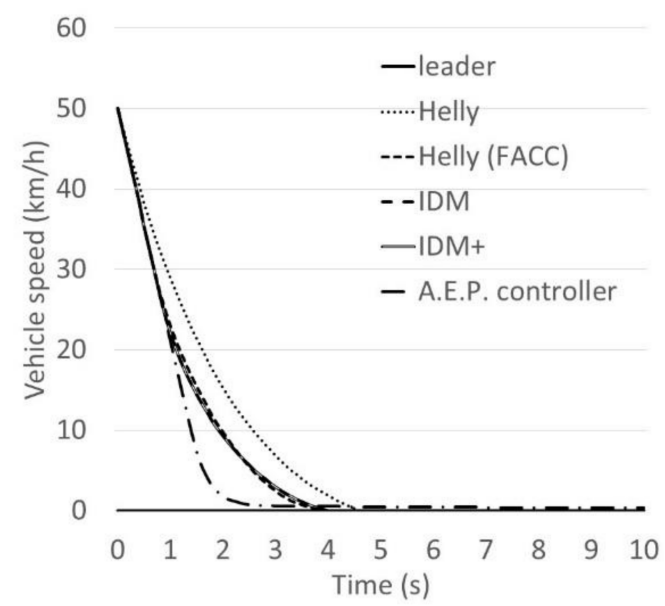

Figure 11. The following vehicle's speed transition of case No.2.

The modified model performs better. The braking ability of the Helly (FACC) model is comparable to that of IDM or IDM+. It should be noted here, that the A.E.P. controller shows very good performance at reducing speed in an emergency. These results are very valuable not only for traffic modelling and accurate prediction of AV behaviour, but also for environmental benefits since there will be benefits in controlling the speed, acceleration, deceleration and savings regarding fuel consumption [22-26]. 


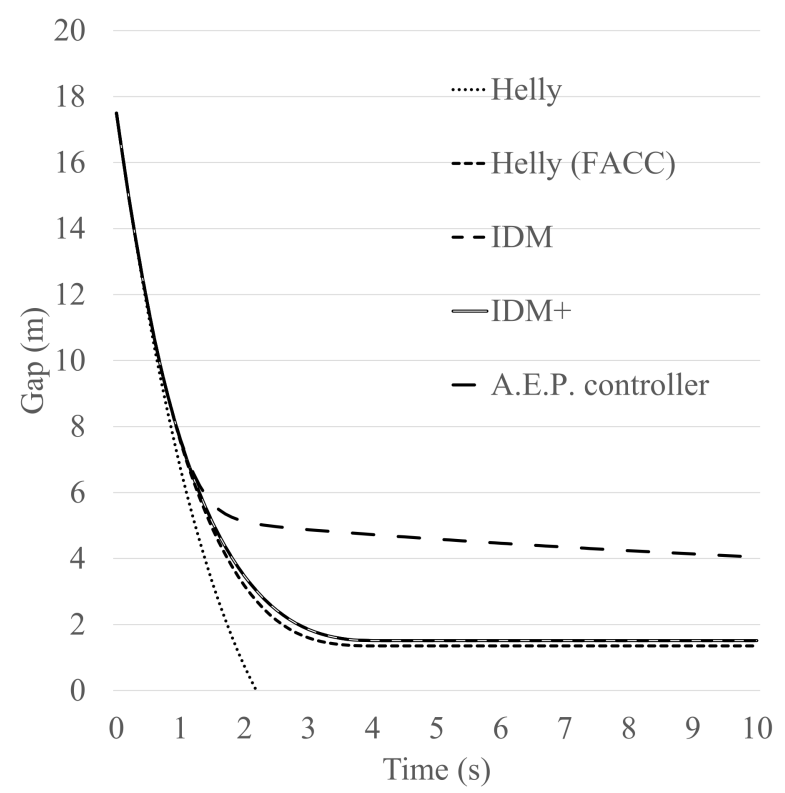

Figure 12. The following vehicle's gap transition of case No.2.

\section{Conclusions and Further Research Recommendations}

The study reports on an investigation of the appropriateness of car-following models in replicating the behaviour of autonomous vehicles. Data from vehicles that are equipped with full-range ACC (FACC) and are already commercially available in the market were obtained and utilised in the assessment. The Helly model, which is a very frequently used car-following model, is assessed and examined for appropriateness for update for the purpose of using it to model autonomous vehicles more efficiently. This involved assessing the parameters of the model and investigating a possible update to include a collision avoidance-system component.

An enhanced car-following model, Helly (FACC), is proposed, assessed and validated. The validation included a comparative analysis of the models' performance in two paradigms that are taken from the Japanese collision avoidance-system standards. The two cases are: a paradigm that states that "when the following vehicle approaches at $50 \mathrm{~km} / \mathrm{h}$ to a stationary vehicle, the following vehicle should not collide with the stationary vehicle or the following vehicle's speed should be less than $20 \mathrm{~km} / \mathrm{h}$ if a collision occurs". The second paradigm states that "when the following vehicle approaches at $50 \mathrm{~km} / \mathrm{h}$ to a leading vehicle that is driving at $20 \mathrm{~km} / \mathrm{h}$, no collision should occur". The braking behaviour of five models, the Helly model, Helly (FACC) model, Intelligent Driver Model (IDM), IDM+ and Alexandros E. Papacharalampous's Model (A.E.P.) controllers have been compared and analysed.

The results have been assessed in terms of the following vehicle's speed and gap transition of the two investigated standards of collision avoidance systems. From the results, it appears that four out of the five investigated models and controller meet the standards of collision avoidance systems. One model did not perform as well which is the original Helly model. In this case, the Helly model shows a collision rather than maintaining a safe gap between the leader vehicle and the following vehicle. This is because the Helly model does not have a collision avoidance function within it.

The modified model performs better as a result of having a braking function. The performance of the Helly (FACC) model is found to be comparable to that of IDM or IDM+. The results also show that the A.E.P. controller performs significantly better than the other models. These results are very valuable not only for traffic modelling and accurate prediction of AV behaviour, but also for the environment, since there will be benefits to controlling the speed, acceleration and deceleration, and savings in fuel consumptions. 
There has been intense progress in ITS, vehicle and information technologies over the past few decades. Many research studies have been undertaken to investigate impacts of such technologies on driving and traffic behaviour. However, the full impact of this technology is yet to be discovered. This is because the technology is not widely available yet and further information is needed for full realisation of its effects. Most of the studies are implemented using simulations or surveys, rather than monitoring actual behaviour. Social and technological issues and predictions of the market have to be further investigated and documented. Further research is typically needed in all these areas. Further areas that are also suggested for future research include the impacts of using technological solutions and autodriving on fuel saving and environmental benefits. In order to enumerate the extent of the validity of the results and representativity to real data, future work should also attempt to compare the results obtained from simulations with more real-world data. $\mathrm{AV}$ are becoming a reality, and very soon, data on these types of vehicles will become more available. Therefore, there will be more chances for further, interesting research.

Author Contributions: Conceptualization, W.S. and A.H.; methodology, W.S. and A.H.; software, A.H. and W.S.; validation, A.H. and W.S.; formal analysis, A.H. and W.S.; investigation, W.S. and A.H.; writing-original draft preparation, A.H. and W.S. writing-review and editing, W.S. and A.H.; visualization, W.S. and A.H.; All authors have read and agreed to the published version of the manuscript.

Funding: This research was funded by the Deanship of Scientific Research at Princess Nourah bint Abdulrahman University through the Fast-track Research Funding Program. The work reported in this article has been conducted while the researcher is affiliated with Princess Nourah bint Abdulrahman University.

Institutional Review Board Statement: Not applicable.

Informed Consent Statement: Not applicable.

Data Availability Statement: Not applicable.

Conflicts of Interest: The authors declare no conflict of interest.

\section{Nomenclature}

$\begin{array}{ll}\text { AV } & \text { Autonomous Vehicles } \\ \text { A.E.P Model } & \text { Alexandros E. Papacharalampous's Model } \\ \text { CACC } & \text { Connected Auto Cruising Control } \\ \text { FACC } & \text { Full-range Auto Cruising Control } \\ \text { GM } & \text { Gipps Model } \\ \text { Helly (FACC) } & \text { Enhanced Helly car-following model } \\ \text { IDM } & \text { Intelligent Driver Model } \\ \text { IDM+ } & \text { Intelligent Driver Model+ } \\ \text { ISA } & \text { Intelligent Speed Adaptation } \\ \text { ITS } & \text { Intelligent Transportation Systems } \\ \text { OVM } & \text { Optimal Velocity Model }\end{array}$

\section{References}

1. Costa, C.; Chatzimilioudis, G.; Zeinalipour-Yazti, D.; Mokbel, M.F. Towards Real-Time Road Traffic Analytics using Telco Big Data. In Proceedings of the International Workshop on Real-Time Business Intelligence and Analytics, Munich, Germany, 28 August 2017; ACM: New York, NY, USA, 2017; p. 5.

2. Zambrano-Martinez, J.L.; Calafate, C.T.; Soler, D.; Lemus-Zúñiga, L.G.; Cano, J.C.; Manzoni, P.; Gayraud, T. A centralized route-management solution for autonomous vehicles in urban areas. Electronics 2019, 8, 722. [CrossRef]

3. Gazis, D.C.; Herman, R.; Richard, R.W. Rothery: Nonlinear follow-the-leader models of traffic flow. Oper. Res. 1961, 4, 545-567. [CrossRef]

4. Gipps, P.G. A behavioural car following model for computer simulation. Transp. Res. B 1981, 15, 105-111. [CrossRef]

5. Helly, W. Simulation of Bottlenecks in Single Lane Traffic Flow. In Proceedings of the International Symposium on the Theory of Traffic Flow, New York, NY, USA, 7-8 December 1959. 
6. Bando, M.; Hasebe, K.; Nakayama, A.; Shibata, A.; Sugiyama, Y. Dynamical model of traffic congestion and numerical simulation. Phys. Rev. 1995, 51, 1035-1042. [CrossRef] [PubMed]

7. Treiber, M.; Hennecke, A.; Helbing, D. Congested traffic states in empirical observations and microscopic simulations. Phys. Rev. E 2000, 62, 1805-1824. [CrossRef] [PubMed]

8. Schakel, W.J.; van Arem, B.; Netten, B.D. Effects of Cooperative Adaptive Cruise Control on traffic flow stability. In Proceedings of the 13th International IEEE Conference on Intelligent Transportation Systems (ITSC), Funchal, Portugal, 19-22 September 2010; pp. 759-764.

9. Treiber, M.; Helbing, D. Microsimulations of Freeway Traffic Including Control Measures. Automatisierungstechnik 2001, 49, 478-484. [CrossRef]

10. Suzuki, K.; Yamada, K.; Horiguchi, R.; Iwatake, K. Impact Assessment of Future Performance of Adaptive Cruise Control for Congestion Mitigation at Expressway Sag Sections. J. Jpn. Soc. Traffic Eng. 2015, 1, B_60-B_67. (In Japanese)

11. Kesting, A.; Treiber, M.; Schönhof, M.; Helbinga, D. Adaptive cruise control design for active congestion avoidance. Transp. Res. Part C 2008, 16, 668-683. [CrossRef]

12. Arem, B.; Cornelie, J.G.; Van, D.; Visser, R. The impact of Cooperative Adaptive Cruise Control on Traffic-Flow Characteristics. IEEE Trans. Intell. Transp. Syst. (ITSC) 2006, 7, 429-436. [CrossRef]

13. Wang, M.; Daamen, W.; Hoogendoorn, S.P.; van Arem, B. Rolling horizon control framework for driver assistance systems. Part I: Mathematical formulation and non-cooperative systems. Transp. Res. Part C Emerg. Technol. 2014, 40, 271-289. [CrossRef]

14. Papacharalampous, A.E.; Wang, M.; Knoop, V.L.; Ros, B.G.; Takahashi, T.; Sakata, I.; van Arem, B.; Hoogendoorn, S.P. Mitigating Congestion at Sags with Adaptive Cruise Control Systems. In Proceedings of the IEEE 18th International Conference on Intelligent Transportation System, Gran Canaria, Spain, 15-18 September 2015; pp. 2451-2457.

15. Goñi-Ros, B.; Knoop, V.; Takahashi, T.; Sakata, I.; van Arem, B.; Hoogendoorn, S.P. Optimization of traffic flow at freeway sags by controlling the acceleration of vehicles equipped with in-car systems. Transp. Res. Part C Emerg. Technol. 2016, 71, 1-18. [CrossRef]

16. Brackston, M.; McDonald, M. Car-following: A historical review. Transp. Res. Part F 1999, 2, 181-196. [CrossRef]

17. Toyota. Available online: https://newsroom.toyota.co.jp/en/corporate/20546438.html (accessed on 2 September 2021).

18. ZTD (Zen Traffic Data). Available online: https:/ / zen-traffic-data.net/english/ (accessed on 2 September 2021).

19. PTV VISSIM 10 User Manual, VisSim/Analyze User Guide v90. Available online: https://www.dot.ny.gov/main/roundabouts/ files/manual_vissim_410.pdf (accessed on 6 September 2021).

20. Ararat, O.; Kural, E.; Guiven, A.B. Development of a collision warning system for Adaptive Cruise Control vehicles using a comparison analysis of recent algorithms. In Proceedings of the 2006 IEEE Intelligent Vehicles Symposium, Meguro, Japan, 13-15 June 2006.

21. Manjunath, K.G.; Jaisankar, N. A Survey on Rear End Collision Avoidance System for Automobiles. Int. J. Eng. Technol. 2013, 5, 1368-1372.

22. NHTSA (National Highway Traffic Safety Administration). Available online: https:/ /www.nhtsa.gov/ (accessed on 6 September 2021).

23. Higatani, A.; Saleh, W. Investigation of the Adequacy of the Current Available Car Following Models of Modelling Autonomous Vehicle; Working Paper; Transport Research Institute, Edinburgh Napier University: Edinburgh, UK, 2018.

24. He, C.R.; Orosz, G. Saving fuel using wireless vehicle-to-vehicle communication. In Proceedings of the 2017 American Control Conference (ACC), Seattle, WA, USA, 24-26 May 2017; pp. 4946-4951.

25. Anselma, P. Optimization-Driven Powertrain-Oriented Adaptive Cruise Control to Improve Energy Saving and Passenger Comfort. Energies 2021, 14, 2897. [CrossRef]

26. Anselma, P.G.; Belingardi, G. Enhancing Energy Saving Opportunities through Rightsizing of a Battery Electric Vehicle Powertrain for Optimal Cooperative Driving. SAE Int. J. Connect. Autom. Veh. 2020, 3, 71-83. [CrossRef] 